\title{
Assessment of Cardiovascular System Abnormalities in Patients with Advanced Primary Hyperparathyroidism by Detailed Echocardiographic Analysis: A Prospective Study
}

\author{
${ }^{1}$ Anand K Mishra, ${ }^{2}$ Amit Agarwal, ${ }^{3}$ Sudeep Kumar, ${ }^{4}$ SK Mishra
}

\begin{abstract}
Aim: Recognizing the paucity of data regarding the incidence of cardiovascular abnormalities in severe primary hyperparathyroidism (PHPT), a prospective study was done for left ventricular (LV) systolic and diastolic function by echocardiography in patients with hyperparathyroidism (HPT) before surgery.
\end{abstract}

Materials and methods: A total of 18 consecutive patients with HPT and an equal number of age- and sex-matched controls were studied by two-dimensional and Doppler echocardiography.

Results: Patients with HPT had higher systolic and diastolic blood pressures (BPs). Despite severe bone disease, nephropathy, and vitamin $D$ deficiency, none had metastatic calcification in myocardium. There was no significant increase in LV systolic dimensions and volume, but decreased ejection fraction (EF) and significant diastolic dysfunction were observed.

Conclusion: Despite an asymptomatic cardiac status, there were decreased EF and significant diastolic dysfunction. The HPT patients despite having severe disease, did not have metastatic calcification.

Keywords: Cardiovascular risk, Echocardiography, Hyperparathyroidism, Parathyroidectomy.

How to cite this article: Mishra AK, Agarwal A, Kumar S, Mishra SK. Assessment of Cardiovascular System Abnormalities in Patients with Advanced Primary Hyperparathyroidism by Detailed Echocardiographic Analysis: A Prospective Study. World J Endoc Surg 2017;9(2):46-50.

Source of support: Nil

Conflict of interest: None

\section{INTRODUCTION}

In recent decades, PHPT has changed its clinical presentation from a disease with bone and renal involvement

\footnotetext{
${ }^{1}$ Professor and Head, ${ }^{2,4}$ Professor, ${ }^{3}$ Associate Professor

${ }^{1}$ Department of Endocrine Surgery, King George's Medical University, Lucknow, Uttar Pradesh, India

${ }^{2,4}$ Department of Endocrine Surgery, Sanjay Gandhi Postgraduate Institute of Medical Sciences, Lucknow, Uttar Pradesh, India

${ }^{3}$ Department of Cardiology, Sanjay Gandhi Postgraduate Institute of Medical Sciences, Lucknow, Uttar Pradesh, India

Corresponding Author: Amit Agarwal, Professor, Department of Endocrine Surgery, Sanjay Gandhi Postgraduate Institute of Medical Sciences, Lucknow, Uttar Pradesh, India, e-mail: amit@sgpgi.ac.in
}

to a frequently asymptomatic disorder detected on routine biochemistry. Unlike in developed countries, we in India continue to see the advanced form of skeletal and kidney involvement in these patients. Our patients are also younger and vitamin $\mathrm{D}$ deficient. The review of English literature revealed that there are limited data on the incidence of cardiovascular abnormalities in severe HPT and there is sparse evidence of increased risk from cardiovascular disease in HPT.

The first suggestion of a link between HPT and increased cardiovascular risk appeared in $1985 .{ }^{1}$ The HPT has been thought to be associated with increased risks of death from cardiovascular disease,,$^{1-3}$ hypertension, ${ }^{4}$ left ventricular hypertrophy (LVH), ${ }^{5,6}$ calcific deposits (valvular and myocardial calcifications) ${ }_{,}^{6,7}$ impaired vascular reactivity, ${ }^{8-10}$ alterations in cardiac conduction, and impaired glucose metabolism. ${ }^{11}$ The nature of some of these associations is in question, because cure of HPT does not lead to improvement of the cardiovascular disorder, e.g., hypertension. Some authors claim that the increased risk of death would not concern the patients of today, who usually have very mild disease, and they suggest that patient series demonstrating increased mortality from HPT are historical. ${ }^{12}$

This prospective study aims to document the anatomical and functional cardiovascular abnormalities and conclusively assess the role of echocardiography for cardiac evaluation in HPT patients.

\section{MATERIALS AND METHODS}

Eighteen consecutive patients of HPT admitted for surgery after biochemical diagnosis in the Department of Endocrine Surgery between April 2004 and April 2006 were included in the study after obtaining verbal informed consent. The control group consisted of ageand sex-matched healthy hospital staff.

All patients underwent clinical, biochemical, and radiological assessments to establish the diagnosis of HPT. Duration of illness was for 9 months (5-26 months). There were 8 male and 10 female patients and age was $38.61 \pm 12.514$ years (15-64 years). All patients underwent detailed echocardiography preoperatively. Echocardiography included an M-mode, two-dimensional, color 
and Doppler (continuous and pulse wave) examination. Images were obtained on a Hewlett-Packard Sonos 5500 with a $4 \mathrm{MH}$ transducer. Recordings were performed with subjects in the supine or left lateral positions. M-mode tracings were obtained at the level of tips of mitral leaflets in parasternal long axis position and measurements of left ventricular end-systolic dimension (LVESD) and LV end-diastolic dimension (LVEDD) were performed according to the recommendations of the American Society of Echocardiography. The left ventricular enddiastolic and end-systolic volumes (LVEDV, LVESV) were calculated from the apical four-chamber view, using the ellipsoid single-plane algorithm. The end-systolic and end-diastolic frames from five consecutive beats were selected and the endocardial outlines were traced. Mean LV volumes and EF were automatically calculated using in-built software in the echocardiography system. Cardiac output was measured by multiplying heart rate with stroke volume.

The LV diastolic function was assessed by measuring the mitral flow velocity recorded in the apical fourchamber view. The pulse Doppler sample volume was placed in the LV inflow tract at the level of mitral leaflet tips and three consecutive measurements were averaged.

Isovolumic relaxation time (IVRT) was measured in the apical five-chamber view with the sample volume placed between the aorta and mitral valve where the recordings of both valves were taken simultaneously.

\section{Statistical Analysis}

All data are expressed as mean and standard deviation. Student's t-test was used for intergroup comparisons. Pearson correlation coefficient test was used to assess the degree of association between different variables. Comparison was done by analysis of variance to see the significant difference among the groups $(\mathrm{p}<0.05$ taken as significant).

\section{RESULTS}

The study population included 18 patients with HPT (8 males, 10 females). The mean age of the patients was $38.61 \pm 12.514$ years ( $15-64$ years). The mean concentration of hemoglobin in patients was $10.4 \pm 1.8 \mathrm{gm} \%$. None of the patients had any symptoms related to cardiovascular involvement nor were there any abnormal auscultatory findings among them. Seven patients (38.8\%) were found to be hypertensive preoperatively, and they were not on medications. The systolic $(131.33 \pm 11.1$ vs $106.05+5.10 \mathrm{~mm}$ $\mathrm{Hg}, \mathrm{p}<0.001)$ and diastolic BPs $(85.67 \pm 6$ vs $63.16+6.08$, $\mathrm{p}<0.001$ ) were significantly higher among patients with HPT as compared with the corresponding values found in controls, though the absolute values were within the normal limits for this population. Resting heart rates were also higher $(85.21 \pm 9.57$ vs $79.9 \pm 6.14$ beats per minute, $\mathrm{p}=0.02$ ) among the patients as compared with controls. Mean preoperative serum calcium was $12.5 \pm 1.41 \mathrm{mg} / \mathrm{dL}$, while mean serum intact parathyroid hormone (PTH) and total alkaline phosphatase levels were 17-fold and 12-fold higher than normal respectively $(1292.06 \pm 959.097$ $\mathrm{pg} / \mathrm{mL}$ and $1293.72 \pm 1329.638 \mathrm{IU} / \mathrm{L})$. These patients had no obvious bone disease; however, all these patients had changes in hand X-ray. The serum creatinine was $1.289 \pm$ 0.6125 ; however, 7 patients had creatinine levels $>2.5 \mathrm{mg}$. Serum vitamin D (calcidiol) level was $14.8 \pm 16.9 \mathrm{ng} / \mathrm{mL}$ (2.3-74.9). Thirteen patients had serum vitamin D levels less than $15 \mathrm{ng} / \mathrm{mL}$. The mean parathyroid gland weight was $8.12+11.8$ (1-52) gm. Other biochemical parameters are shown in Table 1.

Patients with HPT had significantly higher LVESD and LVEDD (Table 2). In view of the enlarged LV size, patients with HPT had significantly higher LVEDV and LVESV. Though EF was within the normal range, the values were lower among patients with HPT as compared with controls (Table 2). The stroke volume and cardiac output were comparable between the two groups. Differences were observed in almost all diastolic parameters in patients of HPT as compared with controls (Table 3); however, the small sample size could probably account for lack of significant difference. There was no correlation between diastolic parameters and levels of serum calcium, PTH, and 25-hydroxy vitamin D (25-OHD). The various $\mathrm{LV}$ diastolic function parameters are indicated in Table 3.

Peak transmitral early filling (E) velocity was lower and peak atrial filling (A) velocity was higher among the patients with HPT. This was also reflected by the E/A velocity ratio, which was significantly lower among patients as compared with the healthy population. Though E wave velocity time integral (EVTI) was similar

Table 1: Age, BP, and biochemical profile of the HPT patients

\begin{tabular}{ll}
\hline Parameters & Values \\
\hline Age & $38.61 \pm 12.514$ (15-64 years) \\
Males:females & $8: 10$ \\
BP systolic $(\mathrm{mm} \mathrm{Hg})$ & $131.33 \pm 11.355$ \\
BP diastolic $(\mathrm{mm} \mathrm{Hg})$ & $85.67 \pm 6.068$ \\
Hemoglobin $(\mathrm{gm} \%)$ & $10.45 \pm 1.88$ \\
Peak calcium $(\mathrm{mg} / \mathrm{dL})$ & $12.520 \pm 1.4134$ \\
Peak phosphorus $(\mathrm{mg} / \mathrm{dL})$ & $2.539 \pm 0.4616$ \\
Serum creatinine $(\mathrm{mg} / \mathrm{dL})$ & $1.289 \pm 0.6125$ \\
Serum alkaline $\mathrm{phosphatase}(\mathrm{IU} / \mathrm{L})$ & $1293.72 \pm 1329.638$ \\
Vitamin D $(25-\mathrm{OHD})(\mathrm{ng} / \mathrm{mL})$ & $14.9839 \pm 16.93545$ \\
Intact PTH $(\mathrm{pg} / \mathrm{mL})$ & $1292.06 \pm 959.097$ \\
Weight of adenoma $(\mathrm{gm})$ & $8.12 \pm 11.825$ \\
\hline
\end{tabular}


Table 2: Left ventricular systolic parameters in HPT patients and control groups

\begin{tabular}{llll}
\hline Systolic parameter & HPT group & Control group & p-value \\
\hline LVEDD $(\mathrm{mm})$ & $49.7 \pm 4.9$ & $45.6 \pm 5.8$ & 0.1 \\
LVESD $(\mathrm{mm})$ & $28.4 \pm 3.8$ & $26.7 \pm 4.9$ & 0.08 \\
LVEDV $(\mathrm{mL})$ & $94.96 \pm 25.31$ & $88.1 \pm 17.5$ & 0.09 \\
LVESV $(\mathrm{mL})$ & $38.19 \pm 10.7$ & $34.2 \pm 9.2$ & 0.07 \\
EF $(\%)$ & $57.4 \pm 10.1$ & $62.3 \pm 5.2$ & 0.06 \\
Stroke volume $(\mathrm{mL})$ & $56.77 \pm 14.78$ & $54.12 \pm 8.5$ & 0.07 \\
Cardiac output $(\mathrm{l} / \mathrm{mt})$ & $48882.22 \pm 132$ & $4113.12 \pm 119$ & 0.08 \\
LV posterior wall thickness in diastole $(\mathrm{mm})$ & $9.4 \pm 1.55$ & $8.8 \pm 2.9$ & 0.2 \\
Interventricular septum thickness in diastole $(\mathrm{mm})$ & $9.7 \pm 1.55$ & $8.5 \pm 1.7$ & 0.2 \\
\hline
\end{tabular}

Table 3: Left ventricular diastolic parameters in HPT patients and control groups

\begin{tabular}{lllr}
\hline Diastolic parameter & HPT group & Control group & $p$-value \\
\hline Peak early diastolic flow velocity E $(\mathrm{m} / \mathrm{sec})$ & $0.96+0.11$ & $1.15+0.13$ & $<0.001$ \\
Peak late diastolic flow velocity A $(\mathrm{m} / \mathrm{sec})$ & $0.73+0.08$ & $0.68+0.08$ & 0.028 \\
Early diastolic flow acceleration time $(\mathrm{msec})$ & $76.85+11.95$ & $79.75+18.34$ & 0.018 \\
Early diastolic flow deceleration time $(\mathrm{msec})$ & $168.38+53.91$ & $146.15+39.24$ & $<0.001$ \\
Early diastolic flow duration $(\mathrm{msec})$ & $206.44+24.32$ & $224.9+32.5$ & 0.064 \\
Total diastolic flow duration (msec) & $362.76+30.56$ & $410.46+32.36$ & $<0.001$ \\
EF slope (m/sec ${ }^{2}$ ) & $6.09+1.89$ & $6.79+1.66$ & 0.192 \\
PVS (m/sec) & $0.79+0.09$ & $0.77+0.05$ & 0.93 \\
PVD (m/sec) & $0.68+0.06$ & $0.57+0.09$ & 0.005 \\
PVS/PVD & $1.25+0.14$ & $1.34+0.18$ & 0.004 \\
E/A & $1.32+0.16$ & $1.76+0.15$ & $<0.001$ \\
IVRT (m/sec) & $68.65+7.86$ & $59.91+8.13$ & 0.002 \\
EVTI (cm) & $10.38+4.02$ & $13.19+2.48$ & 0.155 \\
AVTI (cm) & $6.79+0.92$ & $5.68+0.96$ & $<0.001$ \\
EVTI/AVTI & $1.89+0.28$ & $2.38+0.32$ & $<0.001$ \\
Late diastolic flow acceleration time (msec) & $57.13+20.15$ & $66.54+15.88$ & 0.001 \\
Late diastolic flow deceleration time (msec) & $92.09+22.47$ & $95.6+24.46$ & 0.606 \\
Late diastolic flow duration (msec) & $95.78+38.85$ & $99.71+39.78$ & 0.566 \\
\hline
\end{tabular}

in the two groups, patients with HPT had a much higher A wave VTI (AVTI) when compared with controls. This led to significantly lesser EVTI/AVTI values in patients with HPT.

Eat, Aat, and E duration were significantly lower and Edt was significantly longer among patients as compared with controls (Table 3). The IVRT was significantly more prolonged in patients with HPT, though the absolute mean values were still in the normal range. Though pulmonary vein systolic (PVS) peak velocities were comparable in the two groups, pulmonary vein diastolic (PVD) peak velocities were significantly higher among patients, thus leading to much lower PVS/PVD ratios in the patient population.

None of the patients had aortic or mitral valve involvement or valvular and myocardial calcifications. The mean left atrial size $(37.3 \pm 5.1$ vs $37.4 \pm 4.9 \mathrm{~mm}, \mathrm{p}=0.9)$ and mean aortic dimension $(27.1 \pm 3$ vs $24.9 \pm 5.1 \mathrm{~mm}, \mathrm{p}=0.4)$ were also comparable between the two groups. Lack of any significant aortic valvular involvement was also borne out by the fact that the mean aortic annulus $(22.8 \pm 2.5 \mathrm{vs}$
$21.9 \pm 4.1 \mathrm{~mm})$, mean aortic velocity $(1.29 \pm 0.2$ vs $1.17 \pm 0.2$ $\mathrm{cm} / \mathrm{sec})$, and mean aortic VTI $(23.1 \pm 5.7$ vs $22.7 \pm 5.2 \mathrm{~cm})$ were not significantly different among the two groups.

\section{DISCUSSION}

The first suggestion of a link between HPT and increased cardiovascular risk appeared in 1985. ${ }^{1}$ Several studies done between the 1950s and 1980s attributed the increased mortality in HPT patients to cardiovascular disease. ${ }^{1-11}$ However, due to change in the presentation of HPT to a more milder or asymptomatic disease, subsequent studies refuted the claim. ${ }^{12}$ In India ${ }^{13}$ and other developing countries, the severe form of HPT is still seen. In the present study, all patients had severe disease as evident from Table 1 . We have documented the echocardiographic findings utilizing the latest generation machine to document the cardiovascular functions and myocardial or valvular calcifications.

The BP has been found to be elevated in HPT, but the causes are controversial. Potential mechanisms producing hypertension $(\mathrm{BP}>140 / 90 \mathrm{~mm} \mathrm{Hg}$ ) include 
increased blood calcium levels, elevated intracellular calcium, increased PTH, raised plasma renin activity, and hypomagnesemia. Our study demonstrated that patients had significantly higher systolic and diastolic BPs as compared with controls. Since hypertension is an important cause of cardiac mortality in adults with HPT, our finding of HPT patients having higher BPs as compared with controls, despite being in the normal range, assumes importance. Gennari et al ${ }^{4}$ studied 34 patients with HPT due to a parathyroid adenoma. Ten patients were hypertensive. Before parathyroidectomy, no significant difference was observed between normotensive and hypertensive patients in circulating levels of calcium, intact immunoreactive PTH, and daily total urinary catecholamine excretion. In contrast, plasma renin activity and plasma aldosterone levels were higher in hypertensive patients. After surgery, serum calcium and intact PTH levels were reduced to normal in all patients, while BP, plasma renin activity, and plasma aldosterone levels became normal in 8 of 10 hypertensive patients. These results are consistent with the hypothesis of a direct effect of PTH on renin secretion, which could contribute to the pathogenesis of hypertension.

The LVH has been found to be a strong predictor of cardiovascular morbidity and mortality. ${ }^{14-17}$ A high prevalence of $\mathrm{LVH}(>60 \%)$ has been reported by some authors in both hypertensive and normotensive HPT patients. ${ }^{6,7}$ Also, recently, it has been shown that increased large artery stiffness is an important contributor to the development of cardiovascular disease. ${ }^{18,19}$ In our study, 5 patients $(27 \%)$ had LVH and two out of these were normotensive, suggesting that HPT per se may contribute to LVH independent of hypertension. It has been suggested that PTH per se plays an important role in the maintenance or progression of hypertrophy. ${ }^{20}$ However, in our patients, despite very high PTH levels, cardiac hypertrophy was not common.

The LVH is often accompanied by an impaired filling of the heart during diastole. In our study, we documented diastolic function by observing mitral inflow signals (VTIs, acceleration, and deacceleration time, and ratio of these parameters) along with pulmonary flow velocity, which are relatively immune to the filling conditions of the heart. Ours is the first study that documents the diastolic functions utilizing several parameters. Though Stefenelli et $\mathrm{al}^{6}$ found diastolic dysfunction in $69 \%$ of their hypertensive patients as defined by mitral inflow ratio velocity, which may be altered by the age-related impaired filling as the mean age of their patients was 61.2 \pm 13.9 years, we have documented that mitral inflow $\mathrm{E} / \mathrm{A}$ ratio was $1.32+0.16$ compared with $1.76+0.15$ in control group, which is a more sensitive indicator of impaired diastolic function. We have also documented that pulmonary systolic and diastolic filling velocity ratios were comparatively lower in cases of HPT $(1.25+0.14$ vs $1.34+$ $0.18 ; p=0.004)$, signifying stronger correlation with diastolic dysfunction independent of filling conditions of the heart. Since the mean age of our patients is $38.61 \pm$ 12.514 years, it can be safely presumed that impaired filling pattern observed in our patients may not be related to age, but is a direct sequlae of PTH in HPT patients.

The other contentious issue is of valvular and myocardial calcifications in HPT patients. A high prevalence of these calcifications (62-74\%) as detected by echocardiography has been previously reported. ${ }^{7,8}$ Though the mean serum calcium was high $(12.5 \pm 1.41 \mathrm{mg} / \mathrm{dL})$ in our study, none of the patients or control group had aortic or mitral valve involvement or valvular and myocardial calcifications. Stefenelli et $\mathrm{al}^{6}$ reported increased frequency of aortic valve calcifications (patients 63\%, controls 13\%), mitral valve calcifications (patients $49 \%$; controls $15 \%$ ), and myocardial calcification (patients 69\%; controls $17 \%$ ). In their study, mean serum calcium was $12 \mathrm{mg} / \mathrm{dL}$. Niederle et $\mathrm{al}^{7}$ have observed metastatic calcification in the myocardium in $62 \%$ HPT patients by echocardiography. Lack of calcification in our study can be explained by the fact that our patients were younger ( $38.61 \pm 12.51$ years) and we probably had stricter criteria for calcifications than previous studies. Also, we used an echo machine HP 5500, which has a better resolution. In a recent study by Farahnak et al, ${ }^{21,22}$ they concluded that mild HPT patients did not differ from healthy controls for cardiovascular risk and observed no differences in systolic or diastolic functions or in cardiac morphology between the PHPT patients and the age-matched healthy controls. At baseline, the level of 25-OHD was significantly lower in patients in comparison with controls $(40.1 \pm 16.5)$, but still very high from our patients. Another study from Israel ${ }^{22}$ in severe PHPT patients did not find any beneficial effect of parathyroidectomy on cardiovascular risk.

In our series, there was no significant increase in the LV systolic dimensions, volume, and LV EF, but there was a trend of higher values not reaching a significant level. Ishay et $\mathrm{al}^{23}$ evaluated the effect of parathyroidectomy on metabolic abnormalities associated with cardiovascular disease. A total of 34 patients with HPT underwent assessment before and 1 year after successful parathyroidectomy. They measured serum lipids, glucose, insulin, uric acid, calcium, PTH, C-reactive protein, and bone density. They concluded that surgical treatment had no beneficial effect on cardiovascular risk, as assessed by the metabolic syndrome and insulin resistance markers.

In conclusion, the LV systolic function does not seem to be affected by the HPT, whereas there is definite 
association with diastolic functional abnormalities. Ours is the first study on cardiovascular abnormalities in symptomatic severe, young HPTs with evidence of PTH contributing to diastolic dysfunction.

\section{REFERENCES}

1. Ronni-Sivula H. Causes of death in patients previously operated on for primary hyperparathyroidism. Ann Chir Gynaecol 1985;74(1):13-18.

2. Palmér M, Adami HO, Bergström R, Åkerström G, LjunghallS. Mortality after surgery for primary hyperparathyroidism: a follow-up of 441 patients operated on from 1956 to 1979. Surgery 1987 Jul;102(1):1-7.

3. Hedbäck G, Tisell LE, Bengtsson BA, Hedman I, Oden A. Premature death in patients operated on for primary hyperparathyroidism. World J Surg 1990 Nov-Dec;14(6):829-835.

4. Gennari C, Nami R, Gonnelli S. Hypertension and primary hyperparathyroidism: the role of adrenergic and reninangiotensin-aldosterone systems. Miner Electrolyte Metab 1995 Feb;21(1-3):77-81.

5. Dominiczak AF, Lyall F, Morton JJ, Dargie HJ, Boyle IT, Tune TT, Murray G, Semple PF. Blood pressure, left ventricular mass and intracellular calcium in primary hyperparathyroidism. Clin Sci (Lond) 1990 Feb;78(2):127-132.

6. Stefenelli T, Abela C, Frank H, Koller-Strametz J, Globits S, Bergler-Klein J, Niederle B. Cardiac abnormalities in patients with primary hyperparathyroidism: implications for followup. J Clin Endocrinol Metab 1997 Jan;82(1):106-112.

7. Niederle B, Stefenelli T, Glogar D, Woloszczuk W, Roka R, Mayr H. Cardiac calcific deposits in patients with primary hyperparathyroidism: preliminary results of a prospective echocardiographic study. Surgery 1990 Dec;108(6):1052-1056.

8. Celermajer DS, Sorensen KE, Gooch VM, Spiegelhalter DJ, Miller OI, Sullivan ID, Lloyd JK, Deanfield JE. Non-invasive detection of endothelial dysfunction in children and adults at risk of atherosclerosis. Lancet 1992 Nov;340(8828):1111-1115.

9. Joannides R, Haefeli WE, Linder L, Richard V, Bakkali EH, Thuillez C, Lüscher TF. Nitric oxide is responsible for flowdependent dilation of human peripheral conduit arteries in vivo. Circulation 1995 Mar;91(5):1314-1319.

10. Schleiffer R, Xue H, McCarron DA, Bukoski RD. Effect of chronic and subacute parathyroidectomy on blood pressure and resistance artery contractility in the spontaneously hypertensive rat. J Hypertens 1993 Jul;11(7):709-716.

11. Scherrer U, Sartori C. Defective nitric oxide synthesis. A link between metabolic insulin resistance, sympathetic overactivity and cardiovascular morbidity. Eur J Endocrinol 2000 Apr;142(4): 315-323.

12. Silverberg SJ, Bilezikian JP. Evaluation and management of primary hyperparathyroidism - author's response. J Clin Endocrinol Metab 1997 Mar;82(3):983.

13. Mishra SK, Agarwal G, Kar DK, Gupta SK, Mithal A, Rastad J. Unique clinical characteristics of primary hyperparathyroidism in India. Br J Surg 2001 May;88(5):708-714.

14. Levy D, Garrison RJ, Savage DD, Kannel WB, Castelli WP. Prognostic imolications of echocardiographically determined left ventricular mass in the Framingham Heart study. N Eng J Med 1990 May;322(22):1561-1566.

15. Koren MJ, Devereux RB, Casale PN, Savage DD, Laragh JH. Relation of left ventricular mass and geometry to morbidity and mortality in uncomplicated essential hypertension. Ann Inter Med 1991 Mar;114(5):345-352.

16. Frohlich ED, Apstein C, Chobanian AV, Devereux RB, Dustan HP, Dzau V, Fauad-Tarazi F, Horan MJ, Marcus M, Massie B, et al. The heart in hypertension. N Eng J Med 1992 Oct;327(14):998-1008.

17. Ghali JK, Liao Y, Simmons B, Castaner A, Cao G, Cooper RS. The prognostic role of left ventricular hypertrophy in patients with or without coronary artery disease. Ann Intern Med 1992 Nov;117(10):831-836.

18. Smith JC, Page MD, John R, Wheeler MH, Cockcroft JR, Scanlon MF, Davies JS. Augmentation of central arterial pressure in mild hyperparathyroidism. J Clin Endocrinol Metab 2000 Oct; 85(10):3515-3519.

19. Arnett DK, Evans GW, Riley WA. Arterial stiffness: a anew cardiovascular risk factor. Am J Epidemiology 1994 Oct;140(8):669-682.

20. Symons C, Fortune F, Greenbaum RA, Dandona P. Cardiac hypertrophy, hypertrophic cardiomyopathy, and hyperparathyroidism- an association. Br Heart J 1985 Nov;54(5): 539-542.

21. Farahnak P, Lärfars G, Sten-Linder M, Nilson IL. Mild primary hyperparathyroidism: Vitamin D deficiency and Cardiovascular risk markers. J Clin Endocrinol Metab 2011 Jul;96(7):2112-2118.

22. Farahnak P, Ring M, Caidahl K, Farnebo LO, Eriksson MJ, Nilsson IL. Cardiac function in mild primary hyperparathyroidism and the outcome after parathyroidectomy. Eur J Endocrinol 2010 Sep;163(3):461-467.

23. Ishay A, Herer P, Luboshitzky R. Effects of successful parathyroidectomy on metabolic $\mathrm{CV}$ risk factors in patients with severe primary hyperparathyroidism. Endocr Pract 2011 Jul-Aug;17(4):584-590. 